\title{
QUAL O SÍTIO DE REAÇÃO? UM EXPERIMENTO COMPUTACIONAL
}

Sérgio E. Galembeck* e Giovanni F. Caramori

Departamento de Química, Faculdade de Filosofia Ciências e Letras de Ribeirão Preto, Universidade de São Paulo, Av. Bandeirantes, 3900, 14040-901 Ribeirão Preto - SP

Recebido em 21/10/02; aceito em 5/2/03

WHICH IS THE REACTION SITE? A COMPUTATIONAL EXPERIMENT. A computational quantum chemistry experiment is described on the determination of the most reactive atom in a molecule for a reaction. The reaction studied was the $\mathrm{S}_{\mathrm{N}} 2$ of 4(dimethylamino)pyridine and methyl iodide. Several indexes (HOMO coefficent, $(c)$, charges, $(q)$, nucleophilic softness, $\left(s^{+}\right)$, and Fukui index, $\left(f^{+}\right)$) were employed to verify which correctly describe what nitrogen will react. The calculations were made by AM1 and HF/STO-3G methods. The correct reactivity order is only reproduced by $s^{+}$and $f^{+}$. The lack of agreement of FMO based indexes was discussed.

Keywords: organic reactivity; FMO theory; Fukui indices.

\section{INTRODUÇÃo}

Desde os primórdios da química quântica foram propostos vários índices que buscavam indicar qual átomo é mais reativo em uma dada molécula, para uma dada reação. Durante vários anos empregaram-se os índices baseados na Teoria de Orbitais Moleculares de Fronteira, $(\mathrm{FMO})^{1}$, principalmente os coeficientes dos orbitais de fronteira (HOMO ou LUMO) e as cargas atômicas parciais. Quando empregados adequadamente esses índices produzem resultados satisfatórios $^{2,3}$. Mais recentemente, Parr e colaboradores apresentaram uma formulação rigorosa, baseada na Teoria Funcional de Densidade (DFT), de alguns índices propostos por Pearson, como a dureza ("hardness") e macieza ("softness"), ou por Fukui, como os índices que levam o seu nome ${ }^{4-6}$. Esses índices podem ser divididos em índices globais, como a dureza $(\eta)$ e o potencial químico $(\mu)$, e índices locais, como os índices de Fukui locais $\left(f_{k}^{-} \text {e } f_{k}^{+}\right)^{7}$ e as maciezas locais $\left(s_{k}{ }^{-} \text {e } s_{k}{ }^{+}\right)^{8}$. Vários autores têm empregado estes índices e verificado os limites de sua aplicabilidade 9

Recentemente Hull publicou um experimento no qual, a reação $\mathrm{S}_{\mathrm{N}} 2$ da 4-(dimetilamino)piridina (DMAP) com iodeto de metila produzindo 1-metil,4-(dimetilamino)piridina (TMAP) (Esquema 1) é estudada experimentalmente ${ }^{10}$. O caráter nucleofílico do nitrogênio heterocíclico $\left(\mathrm{N}_{1}\right)$ é racionalizado pela análise do potencial eletrostático molecular (MEP) do DMAP, que indica uma região negativa em torno desse átomo. Isso é confirmado pela maior estabilidade desse produto sobre aquele que resultaria da substituição promovida pelo nitrogênio do grupo amina.<smiles>CN(C)c1ccncc1</smiles>

Esquema 1
Nesse artigo apresentamos uma prática computacional na qual são analisados os índices de reatividade baseados nas teorias FMO e DFT do DMAP. Isso permite aos alunos verificar que é possível prever o sítio de reatividade de um dado reagente e analisar os limites de aplicação dos vários índices.

\section{DESCRIÇÃO DO EXPERIMENTO}

Como preparação à prática, os alunos devem ler o artigo de Hull e responder às questões: por quê a substituição ocorrerá no nitrogênio heterocíclico? Por quê o potencial eletrostático negativo encontra-se concentrado neste nitrogênio? Normalmente os alunos concluem que isso se deve ao fato de o DMAP apresentar uma estrutura de ressonância dipolar (Esquema 2), com a carga negativa concentrada nesse nitrogênio.<smiles>CN(C)c1ccncc1</smiles>

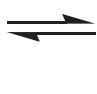<smiles></smiles>

Esquema 2

Após a discussão desses aspectos é feita uma apresentação dos índices de reatividade baseados na teoria FMO e utilizando-se o conceito de dureza e macieza, que já são do conhecimento dos alunos, discutem-se as bases físicas dos índices fundamentados na teoria DFT e as fórmulas para cálculo dos índices de Fukui locais para ataque nucleofílico (Equação 1) e eletrofílico (Equação 2) e os parâmetros de macieza locais (Equação 3) para um átomo $k$ em uma molécula com $N$ elétrons:

$f_{k}^{+}=\left[q_{k}(N+1)+q_{k}(N)\right]$

$f_{k}^{-}=\left[q_{k}(N)+q_{k}(N-1)\right]$

$q_{\mathrm{k}}$ é a carga para o átomo $k$ na molécula em estudo.

$s_{k}^{i}=f_{k}^{i} S \quad$ onde $i=+$ ou -

*e-mail: segalemb@usp.br 
a macieza global, $S$, é dada por

$S=1 /(I E-E A)$

onde $I E$ e $E A$ são a primeira energia de ionização e a afinidade eletrônica da molécula. São discutidos quais os índices que deverão ser utilizados para se determinar o sítio de ataque nucleofílico do DMAP e se conclui que é o átomo com maior coeficiente do HOMO, maior carga negativa e maiores $f_{k}^{+} e s_{k}^{+}$.

A segunda parte da prática envolve o experimento computacional propriamente dito. Os alunos constroem o DMAP no programa Molden ${ }^{11}$. A geometria é otimizada no programa MOPAC $93^{12}$ com o método $\mathrm{AM} 1^{13}$. As cargas atômicas são obtidas pela partição de Mulliken ou pelo ajuste do potencial eletrostático segundo o esquema de Merz-Singh-Kollman (MK) $)^{14,15}$. Os orbitais moleculares são visualizados nos programas Molden ou Molekel ${ }^{16}$. Estes programas são equivalentes tanto na simplicidade do uso, como na qualidade dos gráficos obtidos. Para o cálculo dos índices de Fukui e das maciezas é necessário o cálculo das cargas dos cátions-radicais na geometria do DMAP. Utiliza-se o método ROHF/AM1, para se evitar os problemas de contaminação de spin que surgiriam se fosse utilizado o método irrestrito (UHF).

$\mathrm{O}$ experimento foi planejado para ser concluído em uma aula prática de $2 \mathrm{~h}$, com o uso de microcomputadores com sistema operacional Windows ou UnixPC. No caso de serem realizados cálculos HF/STO-3G, um período adicional de 2 h será necessário.

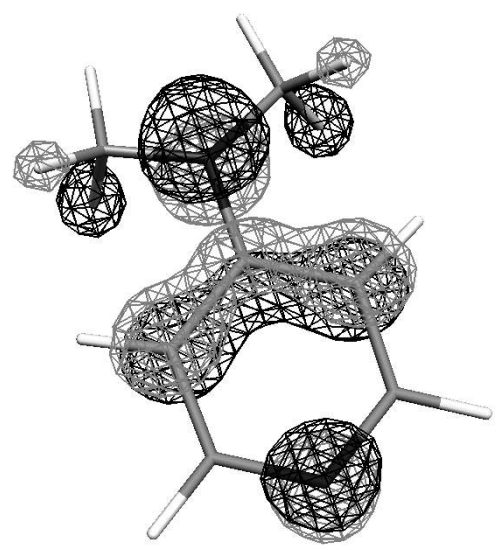

Figura 1. HOMO do DMAP

Tabela 1. Cargas atômicas (q), coeficientes do HOMO (c), índices de Fukui e maciezas nucleofílicas $\left(f^{+} e s^{+}\right)$para os nitrogênios do DMAP. Resultados obtidos pelos métodos AM1 e HF/STO-3G

\begin{tabular}{|c|c|c|c|c|c|}
\hline \multicolumn{6}{|c|}{ AM1 } \\
\hline & \multirow[t]{2}{*}{$\mathrm{c}$} & \multicolumn{2}{|c|}{$\mathrm{q}$} & $f^{+}$ & $s^{+}$ \\
\hline & & Mull $^{\mathrm{a}}$ & MK & Mulla $^{\mathrm{a}}$ MK & Mulla $^{\mathrm{a}}$ MK \\
\hline $\mathrm{N}_{1}$ & 0.344 & -0.219 & -0.403 & $0.025 \quad 0.061$ & $0.084 \quad 0.204$ \\
\hline $\mathrm{N}_{9}$ & 0.654 & -0.366 & -0.437 & $-0.019-0.011$ & $-0.064-0.039$ \\
\hline \multicolumn{6}{|c|}{ HF/STO-3G } \\
\hline & \multirow[t]{2}{*}{$\mathrm{c}$} & \multicolumn{2}{|c|}{$\mathrm{q}$} & $f^{+}$ & $s^{+}$ \\
\hline & & Mull $^{\mathrm{a}}$ & MK & Mulla $^{\mathrm{a}}$ MK & Mulla $^{\mathrm{a}}$ MK \\
\hline $\mathrm{N}_{1}$ & 0.407 & -0.279 & -0.704 & $-0.162-0.145$ & $0.329 \quad 0.294$ \\
\hline $\mathrm{N}_{9}$ & 0.648 & -0.282 & -0.211 & $-0.021-0.052$ & $0.043 \quad 0.087$ \\
\hline
\end{tabular}

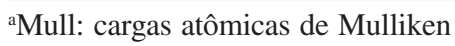

A partir da listagem do cálculo, os alunos obtêm os resultados apresentados na Tabela 1 e com o uso de um programa de visualização o HOMO do DMAP (Figura 1). Por esta tabela e pela figura os alunos concluem que o HOMO e as cargas indicarão que a substituição deverá ser realizada pelo $\mathrm{N}_{9}$, ao contrário do observado experimentalmente. Isso indica o risco do uso indiscriminado desses parâmetros para se indicar o sítio de reatividade. Em contraste, os índices de Fukui e as maciezas indicam corretamente que a substituição se dará pelo $\mathrm{N}_{1}$. O professor deve induzir uma discussão sobre as razões dessa falha, considerando o fato que a maior localização em um dos orbitais de fronteira indica o sítio mais favorável de reação para reagentes moles e a maior carga indica o átomo que sofrerá o ataque de um reagente duro. No caso de reagentes de dureza intermediária, como o iodeto de metila, é difícil se afirmar como será o comportamento desses índices. Além disso, eles freqüentemente não indicam o sítio de reatividade, pois durante o transcurso da reação os reagentes são muito perturbados. Essa perturbação é mais bem refletida pelos índices de Fukui, que são baseados na função de Fukui, $f(r)^{17}$,

$f(r)=\left(\frac{\partial \mu}{\partial v(r)}\right)_{N}=\left(\frac{\partial \varphi(r)}{\partial N}\right)_{v(r)}$

onde $\mathrm{v}(r)$ é o potencial que atua em um elétron devido aos outros núcleos, $N$ é o número de elétrons da molécula, $\varphi(r)$ é a densidade eletrônica e $\mu$ o potencial químico expresso pela relação:

$\mu=\frac{\partial E}{\partial N}=\frac{\partial E}{\partial \varphi(r)}$

onde $E$ é a energia eletrônica total.

O significado físico de $f(r)$ está implícito na sua definição (Equação 5) e é a medida de quão sensível a uma perturbação externa é o potencial químico em um dado ponto, para um número constante de elétrons ou ainda é a variação da densidade eletrônica com o número de elétrons a um potencial externo. Portanto, $f(r)$ dá a reatividade em um dado sítio e pode ser compreendido como um índice de reatividade $^{18}$. Os índices de Fukui locais, $f_{k}^{-}$e $f_{k}^{+}$, (Equações 1-2), são derivados a partir de $f(r)$ pelo método das diferenças finitas. Pelas definições, $f(r), f_{k}^{-}$e $f_{k}^{+}$indicam a perturbação na densidade eletrônica com a variação do número de elétrons que ocorre durante a formação da ligação química. Assim, o átomo com o maior índice de Fukui nucleofílico será o mais reativo frente a uma substituição nucleofílica. Lee e Evans provaram esse conceito analiticamente ${ }^{19}$. A aplicação desse critério ainda é objeto de discussão. Chatarraj, por exemplo, propôs que maior índice de Fukui local indica o átomo mais reativo para reações com reagentes moles e que para reações duro-duro, como reações ácido-base, deve-se usar as cargas atômicas ao invés do índice de Fukui mínimo ${ }^{20}$. Em contraste, a maior maciez local para o ataque eletrofílico, $s_{k}^{-}$, indica o sítio mais básico de fenóis halogenados e, portanto, o sítio de protonação ${ }^{21}$.

Previamente a esse experimento, é desejável que os alunos já tenham tido contato com métodos ab initio e baseados na teoria do funcional de densidade (DFT). Assim, uma pergunta freqüente é se o uso do método Hartree-Fock ou de métodos que incluam a correlação eletrônica deveria incrementar os resultados obtidos nessa prática, especialmente com relação à carga atômica e à maior densidade eletrônica do HOMO. Se houver tempo, os alunos podem realizar cálculos com o método HF/STO-3G. Os resultados são apresentados na Tabela 1 e indicam que apenas as cargas MK e as maciezas apresentam o nitrogênio piridínico, $\mathrm{N}_{1}$, como o melhor nucleófilo para a substituição. As cargas baseadas na definição de Mulliken são 
muito sensíveis às variações de métodos e funções de base, em contraste com aquelas calculadas pelo ajuste do potencial eletrostático ${ }^{21}$. A carga MK foi utilizada para cálculo do sítio de protonação de fenóis halogenados ${ }^{9}$. É curioso notar que, por esse método, o produto mais estável é aquele em que a metila se encontra ligada em $\mathrm{N}_{9}$, $(\Delta \Delta \mathrm{H}=28,8 \mathrm{kcal} / \mathrm{mol})$. Um aumento da função de base para 6-31G(d) não modifica os resultados.

\section{CONCLUSÕES}

Neste experimento computacional, o sítio de substituição nucleofílica da 4-(dimetilamino)piridina foi determinado por parâmetros obtidos pela teoria FMO ou fundamentados na Teoria Funcional de Densidade (DFT), juntamente com o método AM1. Isto permitiu uma análise do campo de aplicação desses índices e de suas limitações. Observou-se que os índices obtidos pela teoria FMO não correspondem aos dados experimentais, em contraste com aqueles obtidos pela DFT. Uma análise qualitativa dos fundamentos de ambos os métodos leva à conclusão da superioridade dos parâmetros obtidos pela DFT, já que eles não são limitados pela dureza do reagente e permitem que se descreva as perturbações que ocorrem durante a reação química. Contudo, o uso do método HF/STO-3G indica que alguns dos índices calculados pela DFT não correspondem à observação experimental, o que indica que os limites da aplicação desses índices ainda não estão claramente definidos.

\section{AGRADECIMENTOS}

Os autores agradecem ao LCCA-USP pela generosa alocação de recursos computacionais. G. F. Caramori agradece à FAPESP por uma bolsa de iniciação científica (processo n 99/08945-5). S. E.
Galembeck agradece ao CNPq (bolsa de produtividade em pesquisa $n^{\circ}$ 301957/88-6), FAPESP e CAPES pelo suporte financeiro.

\section{REFERÊNCIAS}

1. Fleming, I.; Frontier Orbitals and Organic Chemical Reactions, John Wiley and Sons: New York, 1976

2. Armelin, E. A.; Donate, M. P.; Galembeck, S. E.; Tetrahedron 2000, 56, 5105 .

3. Jikyo, T.; Eto, M.; Harano, K.; J. Chem. Soc. 1998, 120, 7841.

4. Parr, R. G.; Pearson, R. G.; J. Am. Chem. Soc. 1983, 105, 7512.

5. Parr, R. G.; Yang, W.; Density Functional Theory of Atoms and Molecules, Oxford University Press: New York, 1989.

6. Duarte, H. A.; Quim. Nova 2001, 24, 501.

7. Parr, R. G.; Yang, W.; J. Am. Chem. Soc. 1984, 106, 4049.

8. Mineva, T.; Neshev, N.; Russo, N.; Silicia, E. Toscano. M.; Adv. Quantum Chem. 1999, 33, 273.

9. Tischenko, O.; Pham-Tran, N-N.; Kryachko, E. S.; Nguyen, M. T.; J. Phys. Chem. A 2001, 105, 8709 e referências citadas neste.

10. Hull, L. A.; J. Chem. Educ. 2001, 78, 420.

11. Schaftenaar, G.; Noordik, J. H.; J. Comput.-Aided Mol. Des. 2000, 14, 123.

12. Stewart, J. J. P.; MOPAC93 (Rev. 2), Fujitsu Ltd.: Tókio, 1995.

13. Dewar, M. J. S.; Zoebisch, G.; Healy, E. F.; J. Am. Chem. Soc. 1985, 107, 3902 .

14. Besler, B. H.; Merz, K. M. Jr.; Kollman, P. A.; J. Comput. Chem. 1990, $11,431$.

15. Singh, U. C.; Kollman, P. A.; J. Comput. Chem. 1984, 5, 129.

16. Flükiger, P.; Lüthi, H. P.; Portmann, S.; Weber, J. Swiss, MOLEKEL 4.1, Center for Scientific Computing: Manno (Switzerland), 2000-2001.

17. Parr, R. G.; Yang, W.; J. Am. Chem. Soc. 1984, 106, 4049.

18. Arulmozhira, S.; Kolandaivel, P.; Mol. Phys. 1997, 90, 55.

19. Li, Y.; Evans, J. N. S.; J. Am. Chem. Soc. 1995, 117, 7756.

20. Chatarraj, P. K.; J. Phys. Chem. A 2001, 105, 511.

21. Proft, F. D.; Martin, J. M. L.; Geerlings, P.; J. Phys. Chem. Lett. 1996, 250, 393. 